\title{
Elevated plasma levels of exosomal BACE1-AS combined with the volume and thickness of the right entorhinal cortex may serve as a biomarker for the detection of Alzheimer's disease
}

\author{
DEWEI WANG ${ }^{1}$, PING WANG $^{1}$, XIANLI BIAN $^{1}$, SHUNLIANG XU $^{1}$, QINGBO ZHOU $^{1}$, YUAN ZHANG $^{2}$, \\ MAO DING ${ }^{1}$, MIN HAN ${ }^{3}$, LING HUANG ${ }^{4}$, JIANZHONG BI ${ }^{1}$, YUXIU JIA ${ }^{5}$ and ZHAOHONG XIE ${ }^{1}$ \\ ${ }^{1}$ Department of Neurology, ${ }^{2}$ Center of Evidence-Based Medicine, ${ }^{3}$ Department of Geriatrics, ${ }^{4}$ Department of Radiology, \\ ${ }^{5}$ Institute of Medical Sciences, The Second Hospital of Shandong University, Jinan, Shandong 250033, P.R. China
}

Received December 06, 2019; Accepted March 30, 2020

DOI: $10.3892 / \mathrm{mmr} .2020 .11118$

\begin{abstract}
Long non-coding RNA (lncRNA) and exosomes are involved in the pathological process of Alzheimer's disease (AD), the pathological changes of which are usually first observed in the entorhinal cortex and hippocampus. The aim of the present study was to determine whether the measurement of plasma exosomal lncRNA combined with image data of the entorhinal cortex and hippocampus could be used as a biomarker of $\mathrm{AD}$. A total of 72 patients with $\mathrm{AD}$ and 62 controls were recruited, and the expression levels of several lncRNAs were assessed. Of the recruited participants, 22 patients and 26 controls received brain 3D-BRAVO sequence magnetic resonance imaging (MRI) scans, which were analyzed using an automated analysis tool. The plasma exosomal $\beta$-site amyloid precursor protein cleaving enzyme-1-antisense transcript (BACE1-AS) levels in patients with AD were significantly higher compared with the controls $(\mathrm{P}<0.005)$. Receiver operating characteristic curve analysis revealed that the area under the curve (AUC) was 0.761 for BACE1-AS, the sensitivity was $87.5 \%$, and the specificity was $61.3 \%$. Analysis of MRI images indicated that the right entorhinal cortex volume $(\mathrm{P}=0.015)$ and thickness $(\mathrm{P}=0.022)$ in patients with $\mathrm{AD}$ were significantly smaller. The AUC was 0.688 for the right entorhinal cortex volume, with a sensitivity of $59.1 \%$, and the specificity was $84.6 \%$. The AUC was 0.689 for right entorhinal cortex
\end{abstract}

Correspondence to: Dr Yuxiu Jia, Institute of Medical Sciences, The Second Hospital of Shandong University, 247 Beiyuan Road, Jinan, Shandong 250033, P.R. China

E-mail: xxyy0307@163.com

Dr Zhaohong Xie, Department of Neurology, The Second Hospital of Shandong University, 247 Beiyuan Road, Jinan, Shandong 250033, P.R. China

E-mail: xie_zhaohong@126.com

Key words: Alzheimer's disease, exosome, $\beta$-site amyloid precursor protein cleaving enzyme-1-antisense transcript, biomarker, entorhinal cortex thickness, with a sensitivity of $80.8 \%$, and the specificity was $59.1 \%$. A series-parallel test which integrated the BACE1-AS with the right entorhinal cortex volume and thickness, raised the specificity and sensitivity to 96.15 and $90.91 \%$, respectively. A logistic regression model demonstrated that combination of the 3 indices provided improved sensitivity and specificity simultaneously, particularly when adjusting for age and sex (AUC, 0.819; sensitivity, 81\%; specificity, 73.1\%). The results of the present study demonstrated that detection of plasma exosomal BACE1-AS levels combined with the volume and thickness of the right entorhinal cortex may be used as a novel biomarker of AD.

\section{Introduction}

As the most well-known form of neurodegenerative diseases, Alzheimer's disease (AD) destroys neurons and disrupts transmission of signals which are required for memories to form and be processed; there are currently 50 million patients who suffer from dementia worldwide $(1,2)$. Although there are numerous studies (3-6) on peripheral biomarkers for AD, suitable peripheral markers present in the blood have not been identified (7).

Exosomes are a type of extracellular vesicle with a diameter of 30-150 nm, which contain several nucleic acids and proteins and are released by almost all types of cells (8). The substances present in exosomes are not easily digested by enzymes as they are protected by lipid membrane bilayers $(9,10)$. Due to their small size, exosomes can pass through the blood-brain barrier (BBB) freely (11-13). Exosomes can be used as biomarkers for several neurodegenerative diseases, including Parkinson's disease and prion diseases (14-16). Exosomes have been shown to contain full-length amyloid precursor protein, $\beta$-secretase, $\gamma$-secretase and amyloid (A) $\beta$, which are involved in the pathological process of development of AD (17).

Long noncoding RNAs (lncRNAs) are abundantly expressed in the brain and exhibit tissue specificity (18). Expression of $\beta$-site amyloid precursor protein cleaving enzyme-1-antisense transcript (BACE1-AS) is upregulated in the brains of patient with AD (18-20). Post-mortem studies have demonstrated that high levels and upregulation of brain 
cytoplasmic 200 RNA (BC200) and 51A in patients with AD were specific to the brain regions associated with AD (18-22).

The atrophy detected by structural magnetic resonance imaging (sMRI) involving the medial temporal lobes, including hippocampus, entorhinal cortex, parahippocampal gyrus and subiculum and amygdala, has been used as a criterion to support a diagnosis of AD. The sensitivities are 70-92\% and specificities are $80-95 \%$ in diagnosing AD using sMRI analysis (23-30).

Based on previous research $(16,18,19)$, it was hypothesized that plasma exosomal lncRNA may be used as a biomarker of AD. As such, the relative expression levels of several lncRNAs from plasma exosomes that are associated with AD were assessed, with the aim of the identifying promising peripheral blood biomarkers for AD. Additionally, the volume of the hippocampus and four parameters of entorhinal cortex were measured using sMRI; entorhinal cortex volume, mean thickness, surface area and mean curvature, and combined with exosomal lncRNAs to improve the sensitivity and specificity of AD prediction.

\section{Materials and methods}

Participants. A total of 134 Chinese Han participants (72 patients with AD and 62 controls) were recruited from the Second Hospital of Shandong University, Shandong, China between May 2017 and July 2019. The patient's age range was 53 to 86 years, and the ratio of male to female patients was 0.89:1. All subjects received a cranial MRI and/or CT scan in order to assess the degree of brain atrophy and to exclude patients with other diseases which may cause cognitive impairment, including acute cerebrovascular disease, hydrocephalus and brain tumors. No cerebrospinal fluid (CSF) samples were tested.

All participants underwent neuropsychological tests including the Montreal Cognitive Assessment Beijing Version $(31,32)$, the Chinese version of the Mini Mental State Examination (MMSE) (33), the Clinical Dementia Rating (CDR) scale (34), the Activities of Daily Living scale (35), and the Hamilton Depression (HAMD) scale (36). Probable AD dementia was diagnosed according to the National Institute on Aging and the Alzheimer's Association criteria (2011) (23). Participants were excluded from the study if they had: i) A history of stroke; ii) psychosis; iii) severe depression (HAMD score $\geq 24$ points); iv) other nervous system diseases, such as Lewy body dementia, Parkinson's disease, encephalitis, brain tumors and traumatic brain injury; or v) some systemic diseases, such as thyroid dysfunction, severe anemia, syphilis or HIV infection (37).

The present study was performed in accordance with the recommendations of the Medical Research Ethics Committee of the Second Hospital of Shandong University. Written informed consent was obtained from all controls and guardians of patients with AD.

Plasma collection and exosome isolation. A total of $5 \mathrm{ml}$ whole blood samples were collected from all participants in EDTA tubes and were centrifuged at $3,000 \mathrm{x}$ g at $4^{\circ} \mathrm{C}$ for $15 \mathrm{~min}$ to obtain the platelet-free plasma, which was subsequently aliquoted and stored at $-80^{\circ} \mathrm{C}$. Exosomes were
Table I. Primer sequence for reverse transcription-quantitative PCR.

\begin{tabular}{ll}
\hline Gene & \multicolumn{1}{c}{ Primer sequences $\left(5^{\prime} \rightarrow 3^{\prime}\right)$} \\
\hline$B A C E 1-A S$ & F: CTTGGGCAAACGAAGGTTGG \\
& R: CCCAGAGCCCAGCATCAAAA \\
$51 A$ & F: TGGGAGAGTCAGCATCTTGAAG \\
& R: ACCCTCTCAGTCGTAGAACTTC \\
$B C 200$ & F: CTGGGCAATATAGCGAGAC \\
& R: TGCTTTGAGGGAAGTTACG \\
$B A C E 1 m R N A$ & F: CACTCTGTTCTGGGTGGTCC \\
& R: CATGGGGGATGCTTACCAGG \\
GAPDH & F: ACTTTGGTATCGTGGAAGGACTCAT \\
& R: GTTTTTCTAGACGGCAGGTCAGG
\end{tabular}

F, forward; R, reverse.

isolated from the plasma using the ExoQuick Plasma prep and Exosome Precipitation kit (EXOQ5TM-1; System Biosciences, LLC). The primary experimental steps were: $0.5 \mathrm{ml}$ plasma was added to $4 \mu \mathrm{l}$ thrombin $(611 \mathrm{U} / \mathrm{ml})$ and incubated at room temperature for $5 \mathrm{~min}$ with agitation, then subsequently centrifuged at $14,000 \mathrm{x}$ g for $5 \mathrm{~min}$ at $4^{\circ} \mathrm{C}$. The serum-like supernatant was collected and further centrifuged at $3,000 \mathrm{xg}$ for $15 \mathrm{~min}$ at $4^{\circ} \mathrm{C}$ to remove cells and debris and then the appropriate volume of ExoQuick Exosome Precipitation Solution was added according to the manufacturer's protocol. After refrigerating the mixture overnight at $4^{\circ} \mathrm{C}$, samples were centrifuged at $1,500 \mathrm{x}$ g for $30 \mathrm{~min}$ at $4^{\circ} \mathrm{C}$. The pellets were resuspended in $\mathrm{PBS}$ and stored at $-80^{\circ} \mathrm{C}(38)$.

Western blot analysis. Western blotting was performed to identify the marker proteins of exosomes. Exosome pellets were cleaved using radioimmunoprecipitation assay lysis buffer (Beyotime Institute of Technology) at $4^{\circ} \mathrm{C}$, and protein concentration was determined using a bicinchoninic acid protein assay kit (cat. no. P0012, Beyotime Institute of Technology). Equal quantities of protein $(20 \mu \mathrm{g})$ were loaded onto a $10 \%$ SDS-gel and resolved using SDS-PAGE. Subsequently, the resolved proteins were transferred to PVDF membranes (EMD Millipore) and incubated overnight at $4^{\circ} \mathrm{C}$ with either apoptosis-linked gene 2-interacting protein X (ALIX; 1:5,000, cat. no. ab186429, Abcam) or cluster of differentiation (CD)63 (1:2,000, cat. no. ab217345, Abcam) antibodies. The membranes were incubated for $1 \mathrm{~h}$ at room temperature with the goat anti-rabbit $\operatorname{IgG} /$ horseradish peroxidase $(1: 5,000$, cat. no. ab6721, Abcam). Signals were visualized using chemiluminescence reagent (EMD Millipore) and densitometry analysis was performed using ImageJ version 1.46 (National Institutes of Health) (39).

Transmission electron microscopy (TEM). The fresh exosomes were resuspended in PBS. After placing on copper grids coated with a layer of formvar/carbon, the specimens were fixed with $3 \%$ glutaraldehyde for $5 \mathrm{~min}$ at room temperature. Subsequently, they were stained negatively with $1 \%$ uranyl 
Table II. Demographics and clinical characteristics of all participants.

\begin{tabular}{|c|c|c|c|c|}
\hline Characteristics & All $(n=134)$ & $\mathrm{AD}(\mathrm{n}=72)$ & Control $(n=62)$ & P-value \\
\hline Age, years & $72.9 \pm 8.0$ & $73.7 \pm 7.7$ & $71.8 \pm 8.1$ & $0.172^{\mathrm{a}}$ \\
\hline Sex, female/male & $77 / 57$ & $38 / 34$ & $39 / 23$ & $0.241^{\mathrm{b}}$ \\
\hline Education, years & $5.0(3.8-11.0)$ & $5.0(4.8-11.0)$ & $6.0(3.0-10.5)$ & $0.940^{\mathrm{c}}$ \\
\hline MMSE & $16.5 \pm 8.2$ & $13.7 \pm 6.7$ & $27.2 \pm 2.1$ & $<0.001^{\mathrm{a}}$ \\
\hline CDR & $0 / 1 / 2 / 3(62 / 9 / 36 / 27)$ & $1 / 2 / 3(9 / 36 / 27)$ & $0(62)$ & - \\
\hline Onset time, years & - & $3(2-6.8)$ & - & - \\
\hline Hypertension & $97(72.4 \%)$ & $51(70.8 \%)$ & $46(74.2 \%)$ & $0.673^{\mathrm{b}}$ \\
\hline $\mathrm{FPG}, \mathrm{mmol} / \mathrm{l}$ & $5.2(4.7-6.2)$ & $5.3(4.6-6.7)$ & $5.1(4.8-5.7)$ & $0.564^{\mathrm{c}}$ \\
\hline $\mathrm{HbA} 1 \mathrm{c}, \%$ & $6.76 \pm 1.86$ & $7.09 \pm 2.1$ & $6.3 \pm 1.38$ & $0.132^{\mathrm{a}}$ \\
\hline \multicolumn{5}{|l|}{ Blood lipid level, mmol/l } \\
\hline TG & $0.99(0.78-1.40)$ & $0.91(0.71-1.21)$ & $1.12(0.87-1.58)$ & $0.009^{\mathrm{c}}$ \\
\hline $\mathrm{TC}$ & $4.48 \pm 1.13$ & $4.14 \pm 0.99$ & $4.89 \pm 1.16$ & $0.001^{\mathrm{a}}$ \\
\hline LDL-C & $2.44 \pm 0.79$ & $2.33 \pm 0.74$ & $2.58 \pm 0.83$ & $0.125^{\mathrm{a}}$ \\
\hline HDL-C & $1.38 \pm 0.45$ & $1.28 \pm 0.42$ & $1.50 \pm 0.45$ & $0.016^{\mathrm{a}}$ \\
\hline $\mathrm{HCY}, \mu \mathrm{mol} / 1$ & $20.9 \pm 7.2$ & $21.0 \pm 7.9$ & $20.6 \pm 4.35$ & $0.852^{\mathrm{a}}$ \\
\hline $\mathrm{Hb}$ (female), $\mathrm{g} / \mathrm{l}$ & $120.6 \pm 14.1$ & $117.9 \pm 15.5$ & $124.1 \pm 11.6$ & $0.194^{\mathrm{a}}$ \\
\hline $\mathrm{Hb}$ (male), g/l & $135.2 \pm 16.3$ & $130.9 \pm 17.5$ & $142.4 \pm 11.2$ & $0.032^{\mathrm{a}}$ \\
\hline Abnormal thyroid hormone levels & $45(33.6 \%)$ & $29(40.2 \%)$ & $16(26.0 \%)$ & $0.274^{\mathrm{b}}$ \\
\hline Heart disease & $62(46.3 \%)$ & $41(57 \%)$ & $21(33.9 \%)$ & $0.091^{\mathrm{b}}$ \\
\hline Smoking status & $45(33.6 \%)$ & $22(30.5 \%)$ & $23(37 \%)$ & $0.643^{\mathrm{b}}$ \\
\hline Drinking status & $39(29.1 \%)$ & $19(26.4 \%)$ & $20(32.3 \%)$ & $0.672^{\mathrm{b}}$ \\
\hline History of anesthesia & $50(37.3 \%)$ & $28(38.9 \%)$ & $22(35.5 \%)$ & $0.791^{\mathrm{b}}$ \\
\hline
\end{tabular}

${ }^{\text {aP }}$-values were calculated by Student's t-test; ${ }^{\text {P}} \mathrm{P}$-values were calculated by $\chi^{2}$ test; ${ }^{\mathrm{c}} \mathrm{P}$-values were calculated by Mann-Whitney $\mathrm{U}$ test; $\mathrm{P}<0.05$ was considered to indicate a statistically significant difference. MMSE, Mini Mental State Examination; CDR, Clinical Dementia Rating Scale; FPG, fasting plasma glucose; HbA1c, hemoglobin A1c; TG, triglyceride; TC, total cholesterol; LDL-C, low-density lipoprotein cholesterol; HDL-C, high-density lipoprotein cholesterol; HCY, homocysteine; Hb, hemoglobin.

acetate for $10 \mathrm{~min}$ at room temperature. Finally, the exosomes were observed using a JEOL 1200EX electron microscope at x10,000 magnification (JEOL, Ltd.) at $80 \mathrm{kV}$ as described previously (40).

Nanoparticle analysis. Nanoparticle tracking analysis (NTA) was performed using Nanoparticle Tracking Analysis (ZetaView; Particle Metrix) to measure the size distribution of particles. Samples were diluted in PBS at a ratio of 1:6,000 to achieve a particle count of $1 \times 10^{8}$ particles per $\mathrm{ml}$, the desired concentration. Then, 3 cycles were performed by scanning 11 cell positions for every measurement and capturing 60 frames per position. All pictures were processed and analyzed using the built-in ZetaView Software (41).

Reverse transcription-quantitative polymerase chain reaction $(R T-q P C R)$. Previous studies have demonstrated that expression levels of 1ncRNA BACE1-AS, 51A, BC200 and BACE1 mRNA are upregulated in the brains of patients with AD and may be detected in the peripheral blood $(18,19,22)$. To expand on these studies, the expression levels of these RNAs in peripheral blood exosomes were examined. Of the 134 recruited participants, the expression levels of BACE1-AS were assessed in all subjects, but the expression of BACE1
mRNA, BC200 and 51A were detected in only 123 subjects due to the limitation of blood specimens.

Total RNA was extracted from the plasma exosomes using RNAiso Plus (Takara Bio, Inc.). The RNA was reverse transcribed using PrimeScript ${ }^{\mathrm{TM}} \mathrm{RT}$ reagent kit (Takara Bio, Inc.) in a total volume of $20 \mu \mathrm{l}$ with the following conditions: $15 \mathrm{~min}$ at $37^{\circ} \mathrm{C}$, and $5 \mathrm{sec}$ at $85^{\circ} \mathrm{C}$. The expression of RNA was detected using quantitative PCR using TB Green Premix Ex Taq (Takara Bio, Inc) on a Bio-Rad CFX 96 Real-Time system (Bio-Rad Laboratories, Inc.). All reactions were performed in triplicate. The thermocycling conditions were: $95^{\circ} \mathrm{C}$ for $10 \mathrm{~min}$; followed by 40 cycles of $95^{\circ} \mathrm{C}$ for $15 \mathrm{sec}$ and $60^{\circ} \mathrm{C}$ for $60 \mathrm{sec}$. GAPDH was used as the internal control. The expression of RNA was calculated using the $2^{-\Delta \Delta \mathrm{Cq}}$ method (42). The primer sequences are presented in Table I.

Acquisition of brain images. Of all the participants, there were 22 patients with AD and 26 controls who received brain MRI scans that met the analysis criteria. The 3.0T magnetic resonance (Discovery MR750W; GE Healthcare) was used for image acquisition. T1-weighted MRI scans were acquired using a 3D-BRAin VOlume (BRAVO) sequence with the following parameters: Repetition time $(\mathrm{TR})=8.2$ msec, echo time $(\mathrm{TE})=.2 \mathrm{msec}$, flip angle $=12^{\circ}$, inversion 
A

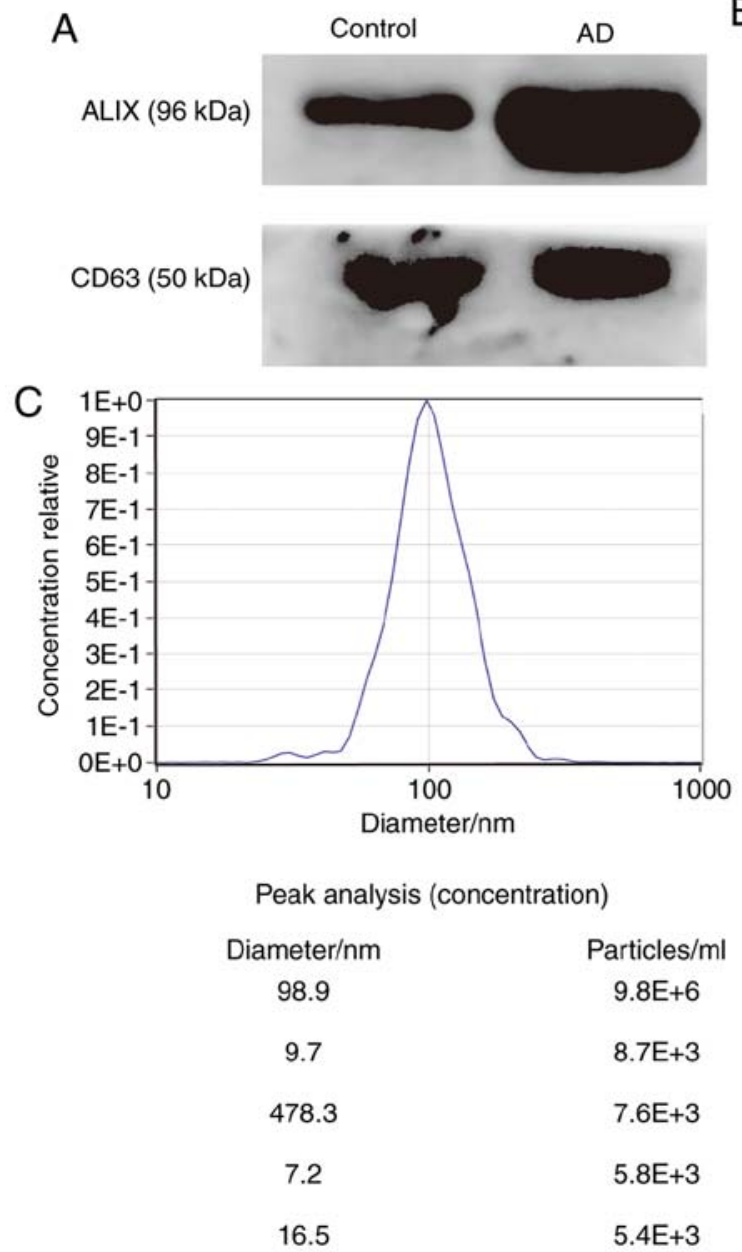

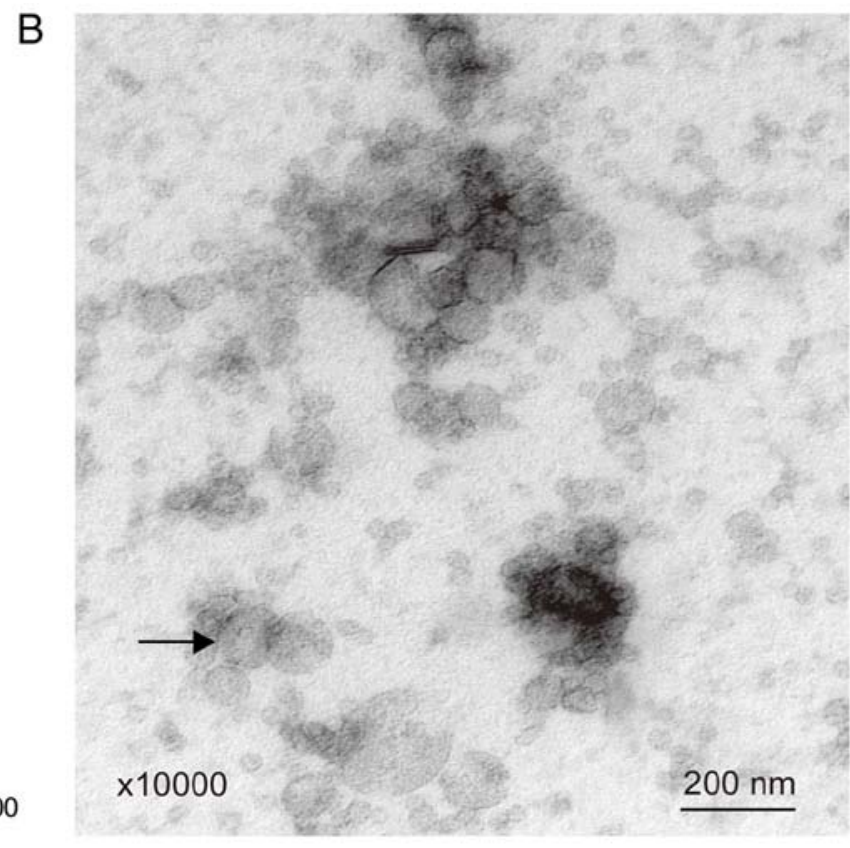

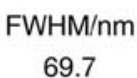

1.9

106.9

2.6

3.4

\section{Percentage \\ 99.7}

0.1

0.1

0.1

0.0

Figure 1. Characterization of plasma exosomes. (A) Western blot analysis of exosomal surface marker proteins (ALIX and CD63). (B) Exosomes exhibited spherical nanoparticles and had a diameter ranging between 30-150 nm under transmission electron microscopy (magnification, $\mathrm{x} 10,000$ ). The arrow shows an exosome that was $100 \mathrm{~nm}$ in diameter. Scale bar=200 nm. (C) Particle size distribution and relative concentration of exosomes measured by Nanoparticle Tracking Analysis. ALIX, apoptosis-linked gene 2-interacting protein X; CD, cluster of differentiation; BACE1-AS, $\beta$-site amyloid precursor protein cleaving enzyme-1-antisense transcript; BC200, brain cytoplasmic 200; FWHM, full width at half maximum; AD, Alzheimer's disease.

time $(\mathrm{TI})=450 \mathrm{msec}$, matrix $=256 \times 256$, slices $=140$, and slice thickness=1.0 mm (43).

Image processing. In order to extract effective analytical features from MR brain T1 structure images, a collaborating company (QED Technique Co., Ltd.) developed a set of automated analysis tools for brain structure for the present study. Hybrid processing algorithms which assisted in the complete removal of non-brain tissue, subcortical volume segmentation and cortical reconstruction without human intervention were used. The specific steps were: i) Tissue segmentation; all MR brain T1 images were registered to a standard ICBM-152 template which then calculated the maximum probabilities and corrected intensity non-uniformities, created an effective brain mask (GM, WM, CSF), and completed tissue segmentation associated with $\mathrm{AD}$, including caudate, putamen, hippocampus, amygdala, ventricles and other regions; ii) cortical morphometric analyses; all T1 spatially normalized images were registered nonlinearly to a surface unbiased group template using surface-based with a spherical warping algorithm. Subsequently, cortical surfaces between WM-GM and GM-CSF were estimated, and then cortical thickness was calculated between these surfaces (WM-GM/GM-CSF). In previous studies, convex (gyri) and concave (sulci) surfaces had different anatomical and connective characteristics; therefore, these units were also analyzed separately to calculate local curvature and surface area. These features were used to distinguish between patients with $\mathrm{AD}$ and control subjects $(44,45)$.

Statistical analysis. Statistical analysis was performed using SPSS Statistics version 24.0 (IBM Corp.) and GraphPad Prism Version 5.0 (GraphPad Software,Inc.). A Kolmogorov-Smirnov or Shapiro-Wilk test was used to check the Gaussian distribution. Gaussian distributed numerical data are presented as the mean \pm standard deviation and skewed distribution variables are expressed as the median and interquartile range. Categorical variable data are expressed as a frequency and percentage. The correlation between lncRNA and clinical features was analyzed using a Spearman's rank or Pearson correlation tests. Logistic regression was used to compute probabilities, the corresponding $95 \%$ confidence interval (CI) and the odds ratio. Quantitative values were compared using a Mann-Whitney U test or Student's t-test. A $\chi^{2}$ test was used to assess differences in categorical data. Receiver operating char- 
Table III. Comparison of plasma RNA expression levels between $\mathrm{AD}$ and controls.

\begin{tabular}{lccr}
\hline RNA & AD & Control & P-value \\
\hline BACE1-AS & $1.36(0.80-2.33)$ & $0.52(0.22-0.96)$ & $<0.005$ \\
BACE1 mRNA & $0.62(0.35-1.53)$ & $0.89(0.34-1.79)$ & 0.327 \\
BC200 & $1.00(0.64-2.55)$ & $1.00(0.43-2.62)$ & 0.354 \\
51A & $1.58(1.09-3.75)$ & $1.44(1.05-2.08)$ & 0.451 \\
\hline
\end{tabular}

AD, Alzheimer's disease; BACE1-AS, $\beta$-site amyloid precursor protein cleaving enzyme- 1 antisense transcript; $\mathrm{BC} 200$, brain cytoplasmic 200 RNA.

acteristic (ROC) curves and the areas under curve (AUC) were used to assess the sensitivity and specificity of the predictive ability of using plasma exosomal lncRNAs and MRI measurement parameters as biomarkers for AD. Multi-index combined detection for diagnosis was based on series-parallel test and logistic regression model. $\mathrm{P}<0.05$ was considered to indicate a statistically significant difference.

\section{Results}

Demographics and clinical features. A total of 72 patients with AD and 62 control subjects were recruited in the present study. The two groups were matched based on sex, age and education level. As shown in Table II, the MMSE scores of patients with AD was significantly lower compared with the controls $(\mathrm{P}<0.001)$. According to the CDR score, there were 9 mild, 36 moderate and 27 severe AD patients. In order to examine potential biomarkers, other clinical characteristics were analyzed, including blood pressure, blood lipid, blood glucose, hemoglobin $(\mathrm{Hb})$, thyroid hormone levels, heart disease, history of anesthesia, and smoking and drinking status. Results demonstrated a significant decrease in triglyceride (TG), total cholesterol (TC), high-density lipoprotein cholesterol (HDL-C) levels, in patients with $\mathrm{AD}$, and in $\mathrm{Hb}$ levels in male patients with $\mathrm{AD}(\mathrm{P}<0.05)$, and none of the other assessed characteristics were significantly different $(\mathrm{P}>0.05)$.

Identification and characterization of plasma exosomes. The exosome marker proteins, ALIX and CD63, were detected using western blotting (Fig. 1A). To further confirm that the sediment extracted from plasma was exosomes, TEM and NTA analyses were performed. Using electron microscopy, it was shown that the vesicles were spherical and had a diameter ranging between $30-150 \mathrm{~nm}$. The arrow in Fig. 1B indicates a vesicle that was $\sim 100 \mathrm{~nm}$ in diameter. The particle diameters ranged between 16.5 and $478.3 \mathrm{~nm}$. The majority of the diameters were $98.9 \mathrm{~nm}$, accounting for $99.7 \%$ of all particles (Fig. 1C). Therefore, the extracellular vesicles extracted matched the characteristics of exosomes (10).

Detection of plasma exosomal lncRNA. The relative expression of lncRNA BACE1-AS, 51A, BC200 and BACE1 mRNA was determined (Table III). As shown in Fig. 2, BACE1-AS levels in patients with AD were significantly higher compared with the controls (Fig. 2A; $\mathrm{P}<0.005$ ). There were no significant differences in the expression levels of BACE1 mRNA (Control, 55; AD, 68; Fig. 2B; $\mathrm{P}=0.327$ ), BC200 (Control, 57; AD, 66; Fig. 2C; $\mathrm{P}=0.354$ ) and 51A (Control, 57; AD, 66; Fig. 2D; $\mathrm{P}=0.451)$ between the two groups.

Correlation of clinical data and exosomal BACE1-AS. As mentioned above, the blood TG, TC and HDL-C levels in patients with AD were significantly lower compared with controls (Table II). In order to determine whether the diagnostic value of BACE1-AS was independent of other clinical characteristics, correlation analyses between BACE1-AS and blood levels of TG, TC and HDL-C and age in both groups, as well as onset time and MMSE scores in patients with AD, was performed. As shown in Fig. 3, there were no significant correlations between these indices and BACE1-AS in both the $\mathrm{AD}$ and control group (Fig. 3A-D, F and $\mathrm{G}$; all $\mathrm{P}>0.05$ ). As previously mentioned, the blood levels of $\mathrm{Hb}$ in male $\mathrm{AD}$ patients were significantly lower compared with the females. The correlation between BACE1-AS and Hb levels in men and women was assessed separately, but no significant correlations were observed (Fig. 3E; P>0.05).

ROC curve analysis of exosomal lncRNAs. As shown in Fig. 2, the BACE1-AS levels of the patients with AD were significantly higher compared with the controls. ROC curve analysis was performed to determine the diagnostic value of BACE1-AS; the AUC was 0.761 (95\% CI, 0.675-0.848; P<0.001). Although there were no statistically significant differences in expression levels of BACE1 mRNA, BC200 or 51A between the two groups, ROC curve analyses were also performed to evaluate their diagnostic value. For 51A, the AUC was 0.540 (95\% CI, 0.436-0.643; $\mathrm{P}=0.451)$, the AUC of $\mathrm{BC} 200$ was 0.541 (95\% CI, $0.436-0.645 ; \mathrm{P}=0.441)$ and the AUC of BACE1 mRNA was 0.540 (95\% CI, 0.437-0.644; $\mathrm{P}=0.444$; Fig. 4).

Acquisition of brain images. There were 22 patients with AD and 26 controls who underwent 3D-BRAVO sequence MRI scans and met the automated analysis criteria. The hippocampus and entorhinal cortex MRI parameters in patients with $\mathrm{AD}$ and in the control group were analyzed, including the bilateral hippocampus volume, entorhinal cortex volume, entorhinal cortex mean thickness, entorhinal cortex surface area and entorhinal cortex mean curvature. As shown in Fig. 5F, the 3 images show the axial, coronal and sagittal position of a brain MRI of a subject from left to right, with the yellow region showing the hippocampus and the green region showing the entorhinal cortex. The results demonstrated that the right entorhinal cortex volume $(\mathrm{P}=0.015)$ and right entorhinal cortex mean thickness $(\mathrm{P}=0.022)$ of patients with $\mathrm{AD}$ were significantly lower compared with the controls (Table IV; Fig. 5A and B). There were no significant correlations between the right entorhinal cortex volume ( $\mathrm{r}=-0.247, \mathrm{P}=0.094)$, mean thickness and BACE1-AS in both groups $(r=-0.246, \mathrm{P}=0.096$; Fig. 5C and D).

To evaluate the potential of the right entorhinal cortex volume and mean thickness as novel biomarkers for AD, ROC curves were plotted (Fig. 5E). The AUC was 0.688 (95\% CI, $0.527-0.850 ; \mathrm{P}=0.015)$ for the right entorhinal cortex volume, the sensitivity was $59.1 \%$, and the specificity was $84.6 \%$ (cutoff 
A

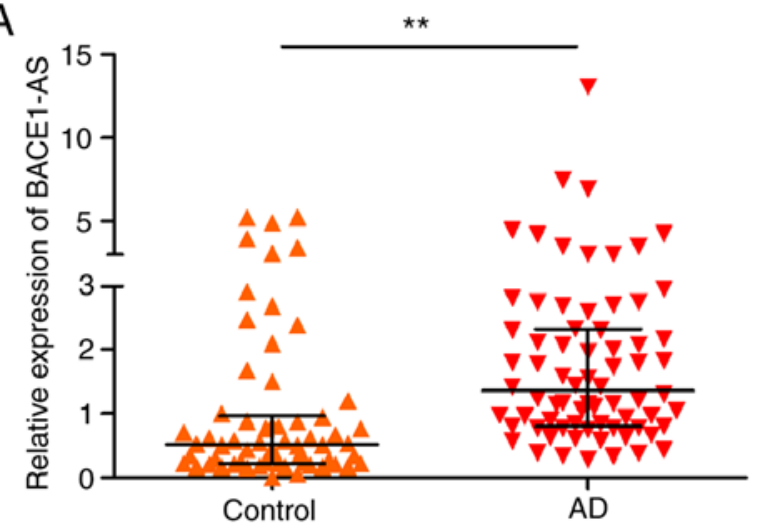

C

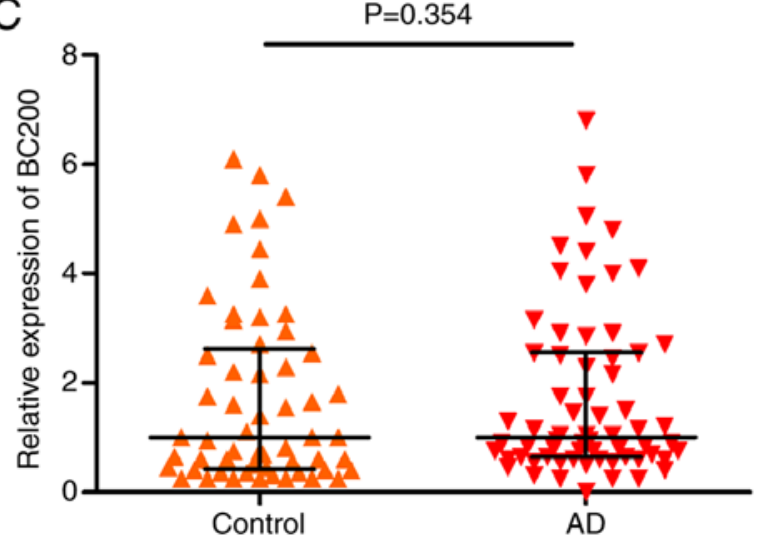

B

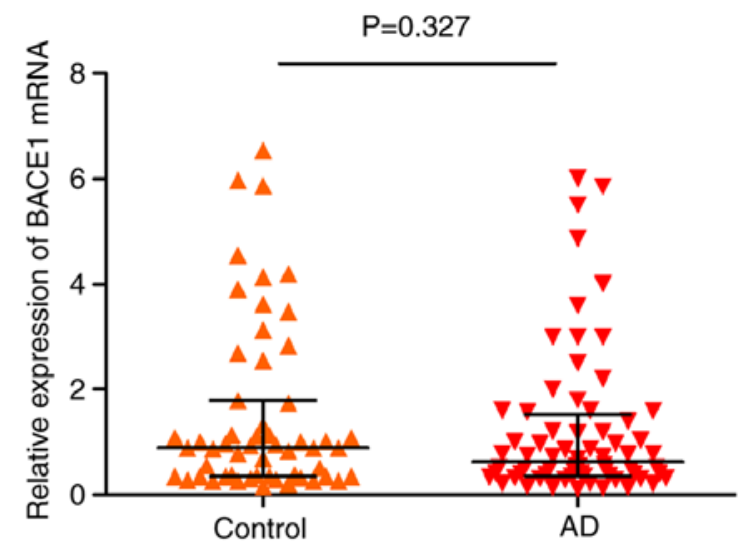

D

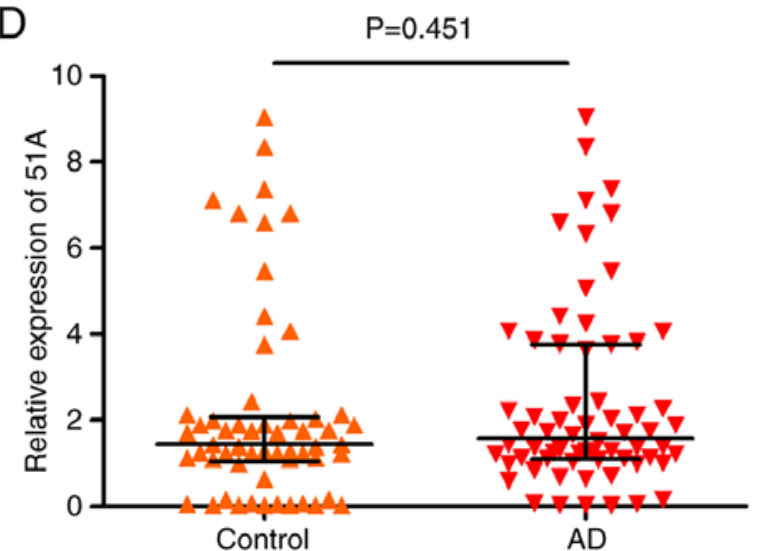

Figure 2. Validation of candidate RNAs (IncRNA BACE1-AS, BC200, 51A and BACE1 mRNA) in plasma exosomes of patients with AD and controls. The expression levels of the 4 RNAs were analyzed by reverse transcription-quantitative polymerase chain reaction and normalized by GAPDH. The bars indicated median with interquartile range. (A) The expression levels of BACE1-AS increased in AD compared with the control group. (B-D) Levels of BACE1 mRNA, $\mathrm{BC} 200$ and 51A had no significant differences between two groups. ${ }^{* *} \mathrm{P}<0.05$ vs. the control group. lnc, long noncoding; BACE1-AS, $\beta$-site amyloid precursor protein cleaving enzyme-1-antisense transcript; BC200, brain cytoplasmic 200; AD, Alzheimer's disease.

point, 1,142.5). The AUC was 0.689 (95\% CI, 0.536-0.843; $\mathrm{P}=0.025)$ for the right entorhinal cortex mean thickness, the sensitivity was $80.8 \%$, and the specificity was $59.1 \%$ (cutoff point, 2.705; Table V).

The diagnostic value of combined detection of BACE1-AS and the two MRI indices. As shown in Table V, none of the 3 indicators (BACE1-AS, right entorhinal cortex volume and mean thickness) had a high sensitivity and specificity at the same time for diagnosis of AD. Therefore, the cumulative performance of the 3 indices together was assessed using a series-parallel test and binary logistic regression models. The series-parallel test of BACE1-AS, right entorhinal cortex volume and right entorhinal cortex mean thickness raised the specificity and sensitivity to 96.15 and $90.91 \%$, respectively. The combination of the 3 indices using a logistic regression model offered improved sensitivity and specificity simultaneously, particularly when adjusting for age and sex (AUC, 0.819; sensitivity, $81 \%$; specificity, $73.1 \%$; accuracy, $76.6 \%$; Table V).

\section{Discussion}

The present study demonstrated that IncRNA BACE1-AS expression levels in plasma exosomes isolated from patients with AD was significantly increased compared with the controls. Furthermore, the diagnostic value of BACE1-AS levels in combination with brain MRI indices were assessed. To the best of the authors' knowledge, the present study is the first to demonstrate that peripheral blood exosomal BACE1-AS levels combined with the volume and thickness of the right entorhinal cortex may serve as a potential biomarker for diagnosis of AD.

The concentrations of $A \beta 42$ and tau protein in CSF and positron emission tomography (PET) imaging are strongly recommended as diagnostic biomarkers for AD (23). However, given the potential trauma caused by lumbar puncture and the high cost of PET, their applications are limited clinically (46). A previous study demonstrated that neuron-derived exosomes in patients with $\mathrm{AD}$ contained $\mathrm{A} \beta$ and tau proteins (47). A recent study demonstrated that the exosomal concentrations of $\mathrm{A} \beta 42$, tau protein phosphorylated at threonine residue 181 (P-T181-tau) and total tau protein (T-tau) were higher in patients with $\mathrm{AD}$ compared with the control group. The levels of A $\beta 42$, P-T181-tau and T-tau in plasma neuronal-derived exosomes were highly correlated with that in the CSF (48). These peripheral blood neuronal-derived exosomal proteins may be used as markers for diagnosis of $\mathrm{AD}$, highlighting the potential of exosomes as biomarkers of AD. In addition to proteins, exosomes contain genetic material, including lncRNAs (10). 

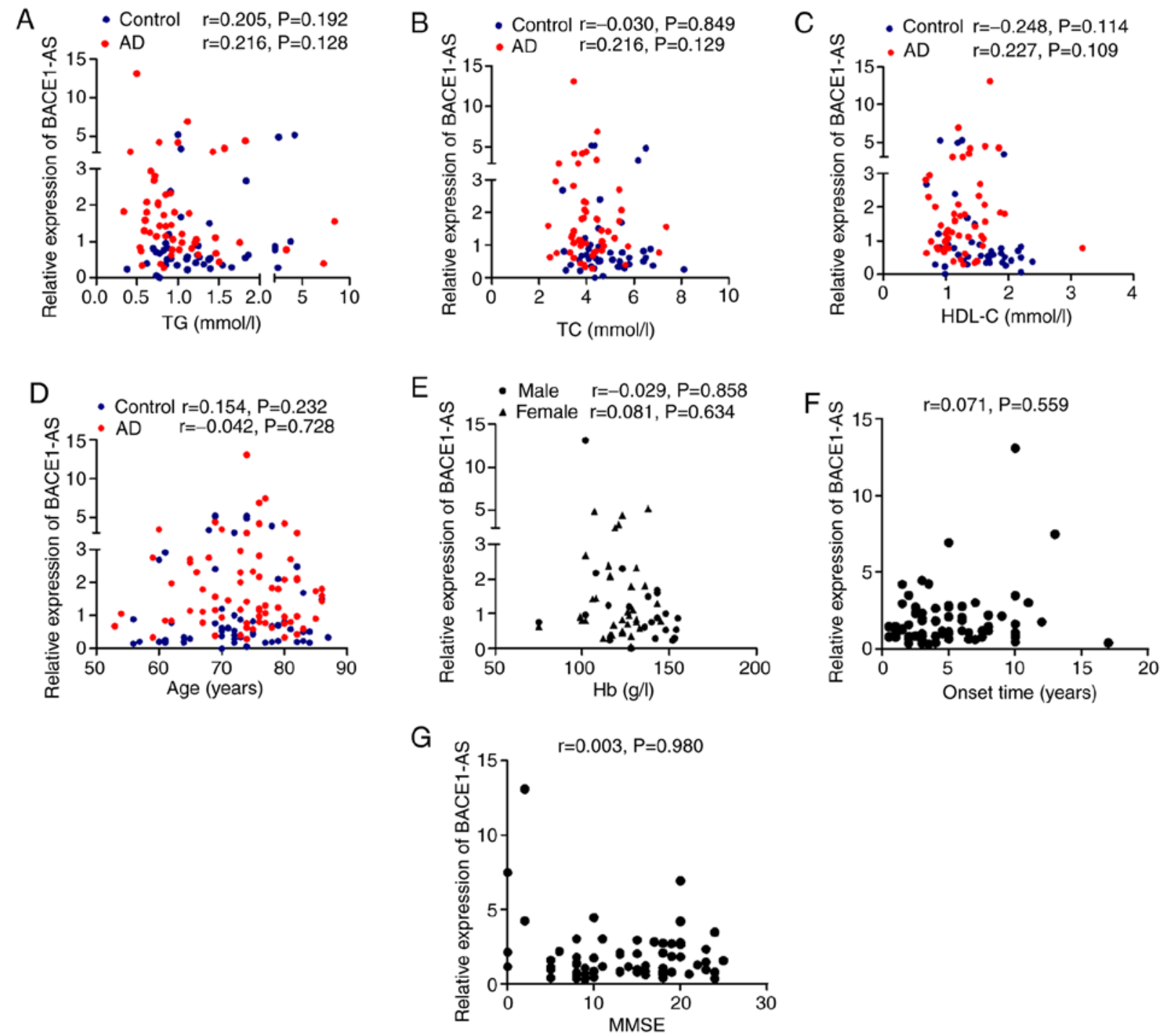

Figure 3. Correlation analyses between expression levels of BACE1-AS and clinical data. P-values and $r$ values are presented in each graph. (A) BACE1-AS vs. blood levels of TG; (B) BACE1-AS vs. blood levels of TC; (C) BACE1-AS vs. blood levels of HDL-C; (D) BACE1-AS vs. age; (E) BACE1-AS vs. blood levels of $\mathrm{Hb}$; (F) BACE1-AS vs. the onset time of AD; (G) BACE1-AS vs. scores of MMSE for AD patients. $\mathrm{P}<0.05$ was considered to indicate a statistically significant difference. There were no significant correlations in all (A-G, $\mathrm{P}>0.05)$. AD, Alzheimer's disease; BACE1-AS, $\beta$-site amyloid precursor protein cleaving enzyme-1-antisense transcript; TG, triglyceride; TC, total cholesterol; HDL-C, high-density lipoprotein cholesterol; Hb, hemoglobin; r, Spearman's rank correlation coefficient; MMSE, Mini Mental State Examination.

Several studies have shown that the expression of lncRNAs, including BACE1-AS, is increased in the brains of patients with AD. BACE1-AS is transcribed from one strand of chromosome 11, which is on the opposite strand to BACE1. BACE1-AS increases BACE1 mRNA stability, which is essential for the production of the toxic $A \beta$ (18-20). Our previous study demonstrated that BACE1-AS was involved in the generation of $A \beta$ and regulation of BACE1 expression in APPsw transgenic cells (49). Therefore, it was hypothesized that BACE1-AS may be a promising biomarker and therapeutic target for diagnosis and treatment of AD. The expression levels of BACE1-AS in human peripheral blood have been examined, but the results are contradictory. Feng et al (50) demonstrated that the expression of BACE1-AS was increased in the plasma of patients with AD. In another study, the expression levels of BACE1-AS were assessed, both in plasma and plasma exosomes, but no significant differences were observed between the patients with $\mathrm{AD}$ and the controls. There was a significant decrease in the levels of BACE1-AS in the plasma of patients who were considered pre-AD (MMSE $\geq 20$ ), and the levels were significantly higher in those considered full-AD (MMSE<20), compared with the controls. Notably, there were no significant differences in BACE1-AS levels in the plasma exosomes between the pre-AD, full-AD and control groups (51).

The present study enrolled more participants than previous studies $(50,51)$. In order to further verify whether BACE1-AS can be used as a biomarker for AD, the BACE1-AS expression levels from plasma exosomes were assessed in patients with $\mathrm{AD}$ and age, sex and education level matched controls. The BACE1-AS levels were significantly higher compared with the control group, but there were no differences in the levels between patients with a varying severity of dementia. The plasma BACE1-AS levels were not simultaneously detected, thus it was not possible to clarify the association between BACE1-AS expression levels in the plasma and exosomes. A previous study demonstrated that exosomes serve a role in 
A

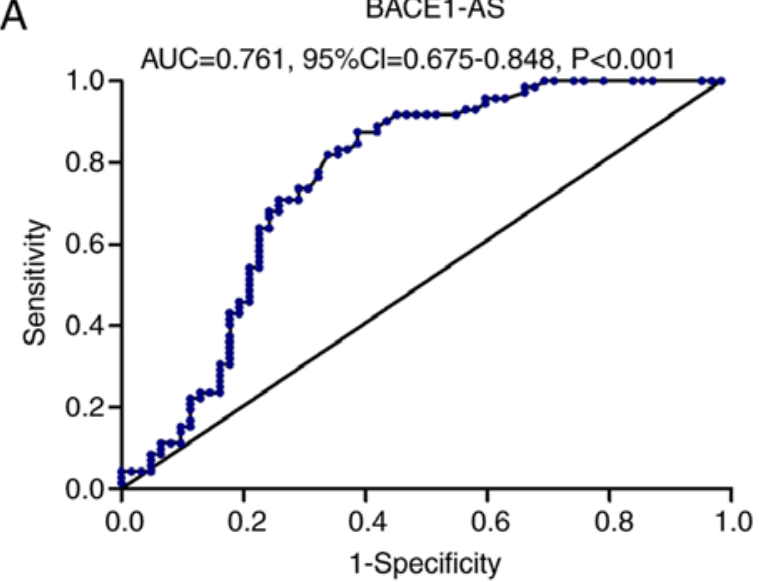

C

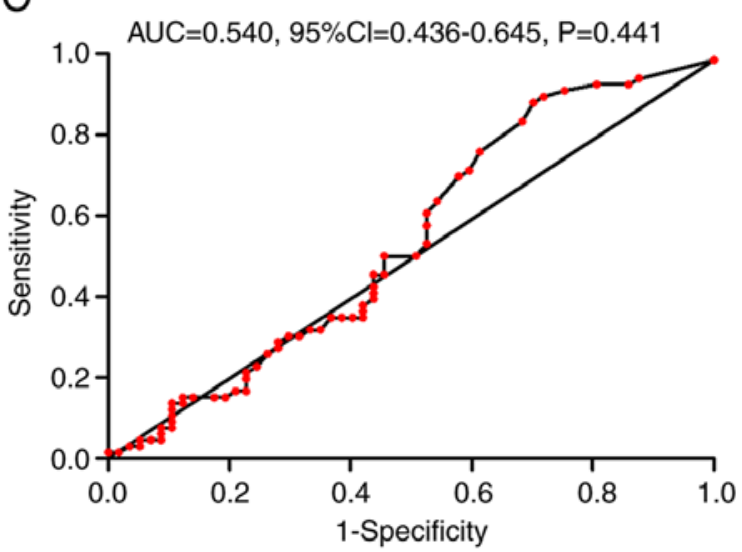

B

BACE1 mRNA

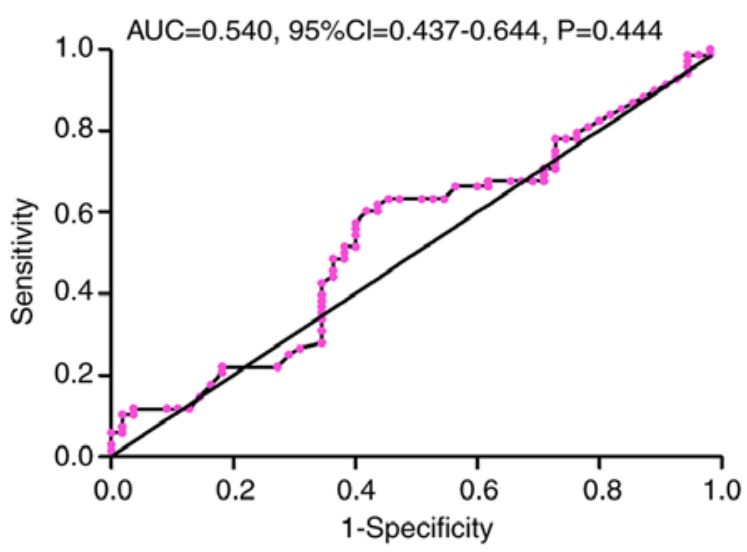

$51 \mathrm{~A}$

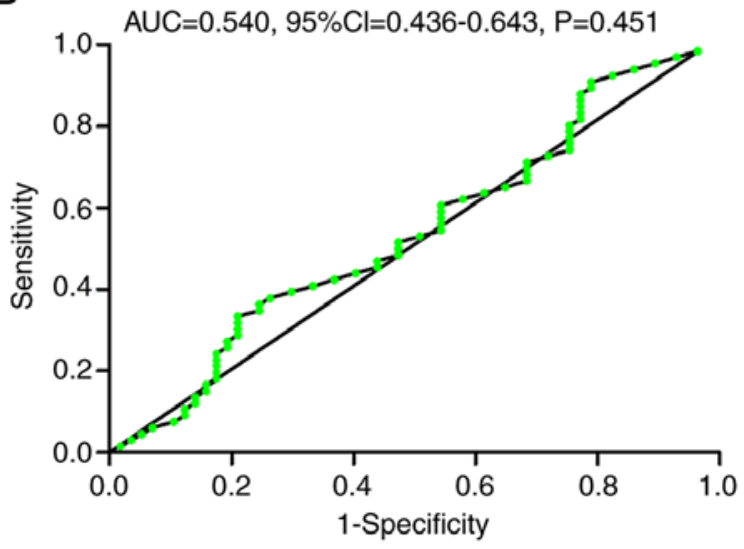

E

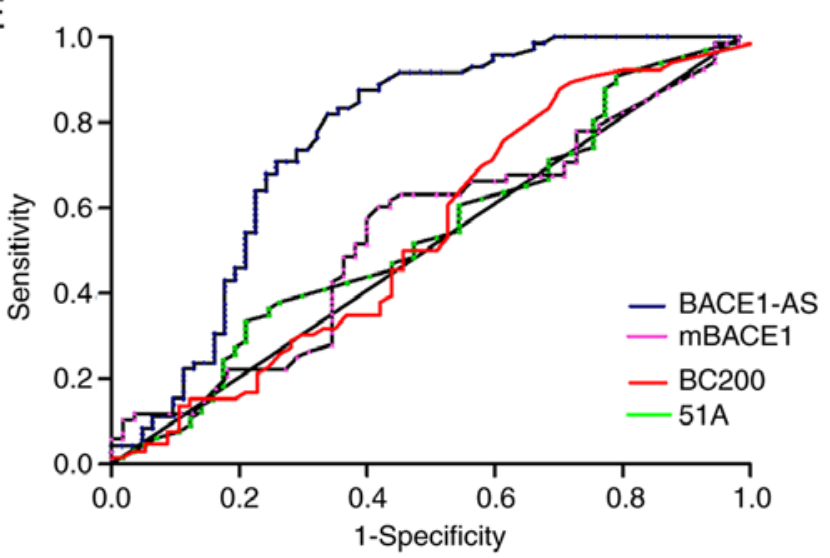

Figure 4. ROC curves of (A) BACE1-AS, (B) BACE1 mRNA, (C) BC200 and (D) 51A for diagnosis of Alzheimer's disease. (E) Combined ROC curves of the four. AUC, $95 \% \mathrm{CI}$ and P-values were presented in graphs. ROC, receiver operating characteristic; BACE1-AS, $\beta$-site amyloid precursor protein cleaving enzyme-1-antisense transcript; BC200, brain cytoplasmic 200; AUC, area under the curve; CI, confidence interval.

communication across the BBB between the periphery and the central nervous system (12). Further research is required to determine whether BACE1-AS crosses the BBB via exosomes.

BACE1-AS affects the expression of $A \beta$ and is involved in the pathogenesis of $\operatorname{AD}(19,49)$; however, whether it is associated with the severity of brain atrophy has not been determined. To examine this, 3D-BRAVO sequence MRI scans of the brains of a portion of the recruited cohort in both groups were taken, and correlation analysis between BACE1-AS and the atrophy of hippocampus and entorhinal cortex was performed. However, the results demonstrated that there was no correlation between these factors. There are two possible reasons for this: i) The cause of AD is complex and abnormal expression of IncRNA is only one of the possible pathogenic mechanisms (18); and ii) 3D-BRAVO sequence required more strict scanning parameters and longer scanning times, and for several patients it was not possible to complete the MRI examination, particularly in patients with severe dementia. Therefore, the MRI parameters of only 48 participants in total were analyzed, which may have made the 
Table IV. Comparison of hippocampus and entorhinal cortex parameters in AD patients and controls.

\begin{tabular}{lccc}
\hline Brain MRI data & AD $(\mathrm{n}=22)$ & Control $(\mathrm{n}=26)$ & P-value \\
\hline Left hippocampus volume, $\mathrm{mm}^{3}$ & $3,666.01 \pm 1,188.36$ & $4,085 \pm 775.80$ & 0.149 \\
Right hippocampus volume, $\mathrm{mm}^{3}$ & $3,926.13 \pm 871.56$ & $4,238.11 \pm 756.22$ & 0.191 \\
Left entorhinal cortex volume, $\mathrm{mm}^{3}$ & $1,354.27 \pm 528.29$ & $1,337.04 \pm 525.24$ & 0.911 \\
Right entorhinal cortex volume, $\mathrm{mm}^{3}$ & $1,114.68 \pm 588.63$ & $1,484.50 \pm 365.55$ & 0.015 \\
Left entorhinal cortex mean thickness, mm & $2.87 \pm 0.45$ & $3.02 \pm 0.43$ & 0.238 \\
Right entorhinal cortex mean thickness, mm & $2.69 \pm 0.44$ & $3.97 \pm 0.38$ & 0.022 \\
Left entorhinal cortex surface area, mm ${ }^{2}$ & $340.18 \pm 125.23$ & $365.08 \pm 117.75$ & 0.960 \\
Right entorhinal cortex surface area, mm $\mathrm{mm}^{2}$ & $293.55 \pm 165.68$ & $0.17 \pm 0.04$ & 0.088 \\
Left entorhinal cortex mean curvature & $0.28 \pm 0.59$ & $0.17 \pm 0.02$ & 0.368 \\
Right entorhinal cortex mean curvature & $0.32 \pm 0.70$ & 0.318
\end{tabular}

P-values were calculated using Student's t-test. AD, Alzheimer's disease.

A

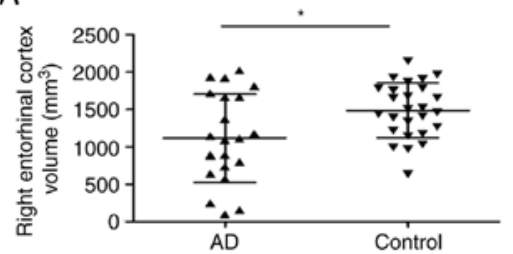

C

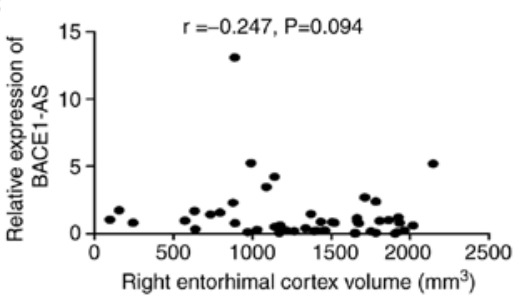

E

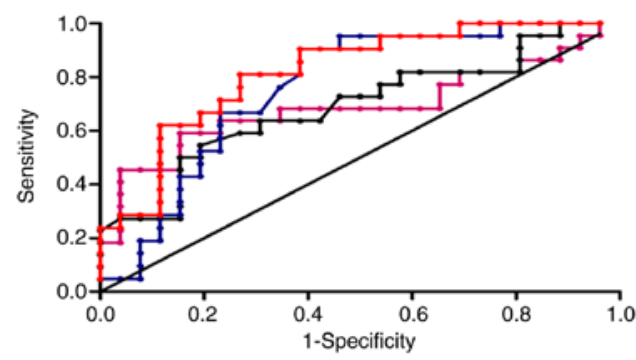

F

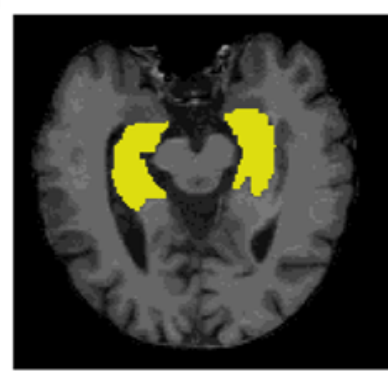

B

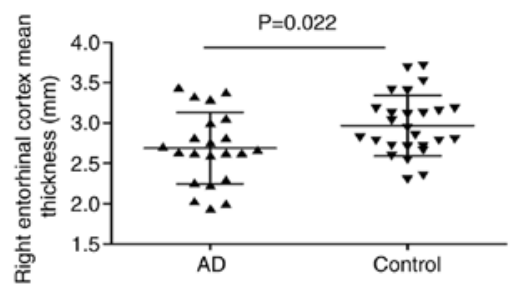

D

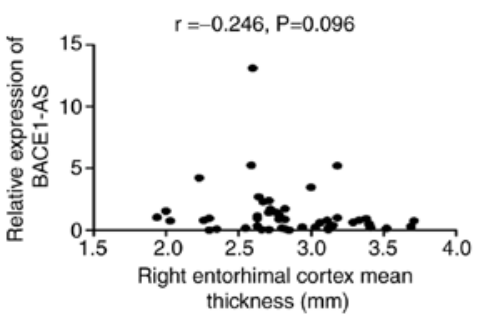

_- Right entorhinal cortex volume Right entorhinal cortex mean thickness

$\rightarrow$ BACE1-AS

Combined diagnostic index (adjusted for age and sex)

- Identity

Figure 5. The diagnostic value of MRI indices and combined detection with BACE1-AS. (A and B) The right entorhinal cortex volume and right entorhinal cortex mean thickness in AD patients were significantly lower than in controls. (C and D) There were no significant correlations between the right entorhinal cortex volume, mean thickness of the right entorhinal cortex and BACE1-AS, respectively, in 48 participants. (E) The ROC curves of right entorhinal cortex volume, right entorhinal cortex mean thickness, BACE1-AS and combined diagnostic index based on a regression model (adjusted age and sex). (F) The axial, coronal and sagittal positions of brain MRI of a subject from left to right, with the yellow area being the hippocampus and the green area being the entorhinal cortex. "P<0.05 vs. the control group. MRI, magnetic resonance imaging; BACE1-AS, $\beta$-site amyloid precursor protein cleaving enzyme-1-antisense transcript; ROC, receiver operating characteristic; AD, Alzheimer's disease. 
Table V. Performance of BACE1-AS, right entorhinal cortex volume and right entorhinal cortex mean thickness in the differential diagnosis of Alzheimer's disease patients from controls.

\begin{tabular}{lccccc}
\hline Diagnostic Indices & AUC & Cutoff point & Sensitivity, $\%$ & Specificity, \% & Accuracy, \% \\
\hline BACE1-AS & 0.761 & 0.623 & 87.5 & 61.3 & 75.37 \\
Right entorhinal cortex volume & 0.688 & $1,142.5$ & 59.1 & 84.6 & 72.92 \\
Right entorhinal cortex & 0.689 & 2.705 & 80.8 & 59.1 & 68.75 \\
mean thickness & & & & & \\
Series test & - & - & 36.36 & 96.15 & 68.75 \\
$\begin{array}{l}\text { Parallel test } \\
\text { Combined diagnostic indices of }\end{array}$ & 0.747 & - & 90.91 & 76.31 & 64.58 \\
$\begin{array}{l}\text { BACE1-AS, right entorhinal } \\
\text { cortex volume and mean thickness }\end{array}$ & & & & & 76.60 \\
Combined diagnostic index of & 0.819 & - & 81 & 73.1 & 76.60 \\
$\begin{array}{l}\text { BACE1-AS, right entorhinal cortex } \\
\text { volume and mean thickness }\end{array}$ & & & & \\
(adjusted for age and sex) & & & & \\
\hline
\end{tabular}

BACE1-AS, $\beta$-site amyloid precursor protein cleaving enzyme-1-antisense transcript.

results biased. Additional participants are required in future studies to further analyze the correlation between BACE1-AS and atrophy of the cortex.

The volume and thickness of the right entorhinal cortex in patients with $\mathrm{AD}$ was significantly lower compared with the control group. ROC curve analysis demonstrated that the sensitivity of the volume of the right entorhinal cortex in the diagnosis of $\mathrm{AD}$ was $59.1 \%$ and the specificity was $84.6 \%$. Regarding the thickness of the right entorhinal cortex, the sensitivity was $80.8 \%$ and the specificity was $59.1 \%$. These results are consistent with those of previous studies reporting the reliability of volumetric imaging of medial temporal lobe structures for the diagnosis of AD (26). Typical AD imaging manifestations observed are temporal lobe atrophy, particularly in the medial temporal lobe, including the hippocampus, parahippocampal gyrus, subiculum, entorhinal cortex and amygdala, which can be used as a diagnostic criterion $(25,30)$. However, there was no difference in the hippocampal volume between patients with $\mathrm{AD}$ and the control group in the present study. A number of studies have demonstrated that hippocampal atrophy can be used as an imaging marker for the diagnosis of $\mathrm{AD}(27,29,37)$; however, the results of the present study contradict this conclusion. This may be due to the insufficient number of cases eligible for imaging analysis. In future studies, the number of participants who undergo 3D-BRAVO sequence MRI scans should be increased to verify the reliability of hippocampal volume as a diagnostic marker of AD.

Although the sensitivity of plasma exosomal BACE1-AS expression levels for the diagnosis of $\mathrm{AD}$ was $87.5 \%$, the specificity was unsatisfactory at $61.3 \%$. Therefore, exosomal BACE1-AS levels and the entorhinal cortex parameters were combined to improve the accuracy of diagnosis of AD. The cumulative performance of the 3 indices was determined using 2 statistical analysis methods; series-parallel test and binary logistic regression models. Through the series-parallel test, the specificity and sensitivity were raised to $96.15 \%$ and
$90.91 \%$, respectively. By applying a logistic regression model, it was shown that the combination of the 3 indices resulted in improved sensitivity and specificity simultaneously, particularly when adjusted for age and sex.

Additionally, other clinical characteristics in both groups were analyzed. Results demonstrated a significant decrease in the levels of TG, TC and HDL-C, and in the Hb levels in males in the plasma of patients with $\mathrm{AD}$, but these were not significantly correlated with BACE1-AS levels. Previous studies have shown that high HDL and TC levels are positively correlated with the risk of $\mathrm{AD}(52,53)$, in contrast to the results of the present study. This may be due to differences in the proportions of patients with moderate to severe dementia in the AD group between the two studies; the severity of $\mathrm{AD}$ increases the risk of malnutrition, leading to a decrease in plasma cholesterol levels $(54,55)$. In addition, the results of the present study demonstrated that $\mathrm{Hb}$ levels in male patients with AD were reduced, consistent with a previous study which suggested that abnormal Hb levels may result in differences in white matter integrity and cerebral perfusion (56).

In the present study, the expression levels of BACE1-AS were assessed in all 134 participants, and the expression of BACE1 mRNA, BC200 and 51A was only detected in 123 subjects. Due to the small quantity of the samples, the expression of the four RNAs was first detected in parts of the samples in order to save the samples, and it was found that only the level of BACE1-AS was different in the two groups. Subsequently, the expression of BACE1-AS was verified in all samples by RT-qPCR. However, some of the remaining sample quantity was too small to complete 3 repeat experiments when detecting other RNAs, so these subjects were excluded.

There were some other certain limitations in the present study. The sample size was small, and more participants are required in future studies to validate the conclusions drawn. 
The present study did not simultaneously detect the plasma total BACE1-AS level and it was uncertain if this lncRNA primarily existed in exosomes in the peripheral blood. Only the total exosomal BACE1-AS levels in plasma were detected; neuron-derived exosomes alone were not detected. Finally, although the MRI parameters of hippocampus and entorhinal cortex were also measured, there was no correlation between BACE1-AS and MRI data. Therefore, determination on whether the abnormal expression of BACE1-AS in the peripheral blood of patients with AD is caused by pathological changes in brain neurons requires further study.

In summary, the present study demonstrated that plasma exosomal BACE1-AS expression levels in patients with AD were significantly higher compared with the controls. Furthermore, BACE1-AS levels combined with right entorhinal cortex MRI parameters may improve the accuracy of diagnosis of AD.

\section{Acknowledgements}

Not applicable.

\section{Funding}

The present study was supported by the National Natural Science Foundation of China (grant nos. 81870848 and 81702538), the Young Talents Fund of the Second Hospital of Shandong University (grant no. 2018YT16), the Fundamental Research Funds of Chinese Academy of Medical Sciences (grant no. 2019-RC-HL-026), the Natural Science Foundation of Shandong Province (grant no. ZR201702160271) and the Shandong University Multidisciplinary Research and Innovation Team of Young Scholars (grant no. 2020QNQT019).

\section{Availability of data and materials}

The datasets used and/or analyzed during the current study are available from the corresponding author on reasonable request.

\section{Authors' contributions}

ZX, YJ, YZ and DW conceived and designed the study, and interpreted the data. DW, XB, MH, MD and $\mathrm{LH}$ performed laboratory experiments. PW, SX, QZ, MH, JB and ZX contributed toward sample collection and processing. DW, XB, and $\mathrm{ZX}$ wrote the manuscript. YZ, JB, YJ and QZ reviewed and edited the manuscript. All authors read and approved the final manuscript.

\section{Ethics approval and consent to participate}

The present study was approved by the Medical Research Ethics Committee of the Second Hospital of Shandong University (approval no. KYLL-2019[LW] 019). All subjects or their guardians provided written informed consent.

\section{Patient consent for publication}

Not applicable.

\section{Competing interests}

The authors declare that they have no competing interests.

\section{References}

1. Bianchi VE, Herrera PF and Laura R: Effect of nutrition on neurodegenerative diseases. A systematic review. Nutr Neurosci 4: 1-25, 2019 (Epub ahead of print).

2. Arvanitakis Z, Shah RC and Bennett DA: Diagnosis and management of dementia: Review. JAMA 322: 1589-1599, 2019.

3. Smedinga M, Tromp K, Schermer MHN and Richard E: Ethical arguments concerning the use of Alzheimer's disease biomarkers in individuals with no or mild cognitive impairment: A systematic review and framework for discussion. J Alzheimers Dis 66: 1309-1322, 2018.

4. Hampel H, O'Bryant SE, Molinuevo JL, Zetterberg H, Masters CL, Lista S, Kiddle SJ, Batrla R and Blennow K: Blood-based biomarkers for Alzheimer disease: Mapping the road to the clinic. Nat Rev Neurol 14: 639-652, 2018.

5. Molinuevo JL, Ayton S, Batrla R, Bednar MM, Bittner T, Cummings J, Fagan AM, Hampel H, Mielke MM, Mikulskis A, et al: Current state of Alzheimer's fluid biomarkers. Acta Neuropathol 136: 821-853, 2018.

6. Li X, Wang H, Long J, Pan G, He T, Anichtchik O, Belshaw R, Albani D, Edison P, Green EK and Scott J: Systematic analysis and biomarker study for Alzheimer's disease. Sci Rep 8: 17394, 2018.

7. Gleerup HS, Hasselbalch SG and Simonsen AH: Biomarkers for Alzheimer's disease in saliva: A systematic review. Dis Markers 2019: 4761054, 2019.

8. Quek C and Hill AF: The role of extracellular vesicles in neurodegenerative diseases. Biochem Biophys Res Commun 483: 1178-1186, 2017.

9. Liu W, Bai X, Zhang A, Huang J, Xu S and Zhang J: Role of exosomes in central nervous system diseases. Front Mol Neurosci 12: 240, 2019.

10. Pegtel DM and Gould SJ: Exosomes. Annu Rev Biochem 88: 487-514, 2019.

11. Zhao Z and Zlokovic BV: Remote control of BBB: A tale of exosomes and microRNA. Cell Res 27: 849-850, 2017.

12. Matsumoto J, Stewart T, Banks WA and Zhang J: The transport mechanism of extracellular vesicles at the blood-brain barrier. Curr Pharm Des 23: 6206-6214, 2017.

13. Yoo YK, Lee J, Kim H, Hwang KS, Yoon DS and Lee JH: Toward exosome-based neuronal diagnostic devices. Micromachines (Basel) 9: E634, 2018.

14. Si X, Tian J, Chen Y, Yan Y, Pu J and Zhang B: Central nervous system-derived exosomal alpha-synuclein in serum may be a biomarker in Parkinson's disease. Neuroscience 413: 308-316, 2019.

15. Hartmann A, Muth C, Dabrowski O, Krasemann S and Glatzel M: Exosomes and the prion protein: More than one truth. Front Neurosci 11: 194, 2017.

16. Yang TT, Liu CG, Gao SC, Zhang Y and Wang PC: The serum exosome derived MicroRNA-135a,-193b, and -384 were potential Alzheimer's disease biomarkers. Biomed Environ Sci 31: 87-96, 2018.

17. Bellingham SA, Guo BB, Coleman BM and Hill AF: Exosomes: Vehicles for the transfer of toxic proteins associated with neurodegenerative diseases. Front Physiol 3: 124, 2012.

18. Cortini F, Roma F and Villa C: Emerging roles of long non-coding RNAs in the pathogenesis of Alzheimer's disease. Ageing Res Rev 50: 19-26, 2019.

19. Luo Q and Chen Y: Long noncoding RNAs and Alzheimer's disease. Clin Interv Aging 11: 867-872, 2016.

20. Faghihi MA, Modarresi F, Khalil AM, Wood DE, Sahagan BG, Morgan TE, Finch CE, St Laurent G 3rd, Kenny PJ and Wahlestedt C: Expression of a noncoding RNA is elevated in Alzheimer's disease and drives rapid feed-forward regulation of beta-secretase. Nat Med 14: 723-730, 2008.

21. Ciarlo E, Massone S, Penna I, Nizzari M, Gigoni A, Dieci G, Russo C, Florio T, Cancedda R and Pagano A: An intronic ncRNA-dependent regulation of SORL1 expression affecting $\mathrm{A} \beta$ formation is upregulated in post-mortem Alzheimer's disease brain samples. Dis Model Mech 6: 424-433, 2013.

22. Mus E, Hof PR and Tiedge H: Dendritic BC200 RNA in aging and in Alzheimer's disease. Proc Natl Acad Sci USA 104: 10679-10684, 2007. 
23. McKhann GM, Knopman DS, Chertkow H, Hyman BT, Jack CR Jr, Kawas CH, Klunk WE, Koroshetz WJ, Manly JJ, Mayeux R, et al: The diagnosis of dementia due to Alzheimer's disease: Recommendations from the national institute on aging-Alzheimer's association workgroups on diagnostic guidelines for Alzheimer's disease. Alzheimers Dement 7 : 263-269, 2011.

24. Jack CR Jr, Bennett DA, Blennow $\mathrm{K}$, Carrillo MC, Dunn B, Haeberlein SB, Holtzman DM, Jagust W, Jessen F, Karlawish J, et al: NIA-AA research framework: Toward a biological definition of Alzheimer's disease. Alzheimers Dement 14: 535-562, 2018.

25. Calvini P, Chincarini A, Gemme G, Penco MA, Squarcia S, Nobili F, Rodriguez G, Bellotti R, Catanzariti E, Cerello P, et al: Automatic analysis of medial temporal lobe atrophy from structural MRIs for the early assessment of Alzheimer disease. Med Phys 36: 3737-3747, 2009.

26. Menéndez-González M, de Celis Alonso B, Salas-Pacheco J and Arias-Carrión O: Structural neuroimaging of the medial temporal lobe in Alzheimer's disease clinical trials. J Alzheimers Dis 48: 581-589, 2015.

27. Juottonen K, Laakso MP, Partanen K and Soininen H: Comparative MR analysis of the entorhinal cortex and hippocampus in diagnosing Alzheimer disease. AJNR Am J Neuroradiol 20: 139-144, 1999.

28. Ou YN, Xu W, Li JQ, Guo Y, Cui M, Chen KL, Huang YY, Dong Q, Tan L and Yu JT; Alzheimer's Disease Neuroimaging Initiative: FDG-PET as an independent biomarker for Alzheimer's biological diagnosis: A longitudinal study. Alzheimers Res Ther 11: 57, 2019.

29. Pennanen C, Kivipelto M, Tuomainen S, Hartikainen $P$, Hänninen T, Laakso MP, Hallikainen M, Vanhanen M, Nissinen A, Helkala EL, et al: Hippocampus and entorhinal cortex in mild cognitive impairment and early AD. Neurobiol Aging 25: 303-310, 2004.

30. Kantarci K: Magnetic resonance markers for early diagnosis and progression of Alzheimer's disease. Expert Rev Neurother 5: 663-670, 2005

31. Yu J, Li J and Huang X: The Beijing version of the montreal cognitive assessment as a brief screening tool for mild cognitive impairment: A community-based study. BMC Psychiatry 12: $156,2012$.

32. Lu J, Li D, Li F, Zhou A, Wang F, Zuo X, Jia XF, Song H and Jia J: Montreal cognitive assessment in detecting cognitive impairment in Chinese elderly individuals: A population-based study. J Geriatr Psychiatry Neurol 24: 184-190, 2011.

33. Yang Z, Holt HK, Fan JH, Ma L, Liu Y, Chen W, Como P, Zhang L and Qiao YL: Optimal cutoff scores for Alzheimer's disease using the Chinese version of mini-mental state examination among Chinese population living in rural areas. Am J Alzheimers Dis Other Demen 31: 650-657, 2016.

34. Morris JC: The clinical dementia rating (CDR): Current version and scoring rules. Neurology 43: 2412-2414, 1993.

35. Juva K, Sulkava R, Erkinjuntti T, Ylikoski R, Valvanne J and Tilvis R: Staging the severity of dementia: Comparison of clinical (CDR, DSM-III-R), functional (ADL, IADL) and cognitive (MMSE) scales. Acta Neurol Scand 90: 293-298, 1994.

36. Mulsant BH, Sweet R, Rifai AH, Pasternak RE, McEachran A and Zubenko GS: The use of the hamilton rating scale for depression in elderly patients with cognitive impairment and physical Illness. Am J Geriatr Psychiatry 2: 220-229, 1994

37. Zhao W, Wang X, Yin C, He M, Li S and Han Y: Trajectories of the hippocampal subfields atrophy in the Alzheimer's disease: A structural imaging study. Front Neuroinform 13: 13, 2019.

38. Zhang X, Lu A, Li Z, Sun J, Dai D and Qian L: Exosomes secreted by endothelial progenitor cells improve the bioactivity of pulmonary microvascular endothelial cells exposed to hyperoxia in vitro. Ann Transl Med 7: 254, 2019.

39. Pua HH, Happ HC, Gray CJ, Mar DJ, Chiou NT, Hesse LE and Ansel KM: Increased hematopoietic extracellular RNAs and vesicles in the lung during allergic airway responses. Cell Rep 26: 933-944.e4, 2019.

40. Hannafon BN, Trigoso YD, Calloway CL, Zhao YD, Lum DH, Welm AL, Zhao ZJ, Blick KE, Dooley WC and Ding WQ: Plasma exosome microRNAs are indicative of breast cancer. Breast Cancer Res 18: 90, 2016.

41. Bachurski D, Schuldner M, Nguyen PH, Malz A, Reiners KS, Grenzi PC, Babatz F, Schauss AC, Hansen HP, Hallek M and Pogge von Strandmann E: Extracellular vesicle measurements with nanoparticle tracking analysis-an accuracy and repeatability comparison between NanoSight NS300 and ZetaView. J Extracell Vesicles 8: 1596016, 2019.
42. Livak KJ and Schmittgen TD: Analysis of relative gene expression data using real-time quantitative PCR and the 2(-Delta Delta C(T)) method. Methods 25: 402-408, 2001.

43. Yang ST, Lee JD, Chang TC, Huang CH, Wang JJ, Hsu WC, Chan HL, Wai YY and Li KY: Discrimination between Alzheimer's disease and mild cognitive impairment using SOM and PSO-SVM. Comput Math Methods Med 2013: 253670, 2013.

44. Pagnozzi AM, Fripp J and Rose SE: Quantifying deep grey matter atrophy using automated segmentation approaches: A systematic review of structural MRI studies. Neuroimage 201: $116018,2019$.

45. Allison SL, Koscik RL, Cary RP, Jonaitis EM, Rowley HA, Chin NA, Zetterberg H, Blennow K, Carlsson CM, Asthana S, et al: Comparison of different MRI-based morphometric estimates for defining neurodegeneration across the Alzheimer's disease continuum. Neuroimage Clin 23: 101895, 2019.

46. Olsson B, Lautner R, Andreasson U, Öhrfelt A, Portelius E, Bjerke M, Hölttä M, Rosén C, Olsson C, Strobel G, et al: CSF and blood biomarkers for the diagnosis of Alzheimer's disease: A systematic review and meta-analysis. Lancet Neurol 15: 673-684, 2016.

47. Fiandaca MS, Kapogiannis D, Mapstone M, Boxer A, Eitan E, Schwartz JB, Abner EL, Petersen RC, Federoff HJ, Miller BL and Goetzl EJ: Identification of preclinical Alzheimer's disease by a profile of pathogenic proteins in neurally derived blood exosomes: A case-control study. Alzheimers Dement 11: 600-607.e1, 2015.

48. Jia L, Qiu Q, Zhang H, Chu L, Du Y, Zhang J, Zhou C, Liang F, Shi S, Wang S, et al: Concordance between the assessment of A $\beta 42$, T-tau, and P-T181-tau in peripheral blood neuronal-derived exosomes and cerebrospinal fluid. Alzheimers Dement 15: 1071-1080, 2019.

49. Li F, Wang Y, Yang H, Xu Y, Zhou X, Zhang X, Xie Z and Bi J: The effect of BACE1-AS on $\beta$-amyloid generation by regulating BACE1 mRNA expression. BMC Mol Biol 20: 23, 2019.

50. Feng L, Liao YT, He JC, Xie CL, Chen SY, Fan HH, Su ZP and Wang Z: Plasma long non-coding RNA BACE1 as a novel biomarker for diagnosis of Alzheimer disease. BMC Neurol 18: 4, 2018.

51. Fotuhi SN, Khalaj-Kondori M, Hoseinpour Feizi MA and Talebi M: Long non-coding RNA BACE1-AS may serve as an Alzheimer's disease blood-based biomarker. J Mol Neurosci 69: 351-359, 2019.

52. Anstey KJ, Ashby-Mitchell K and Peters R: Updating the evidence on the association between serum cholesterol and risk of late-life dementia: Review and meta-analysis. J Alzheimers Dis 56: 215-228, 2017.

53. Espinosa Del Pozo PH, Espinosa PS, Donadi EA, Martinez EZ, Salazar-Uribe JC, Guerrero MA, Moriguti JC, Colcha MC, Garcia SE, Naranjo R, et al: Cognitive decline in adults aged 65 and older in cumbayá, quito, ecuador: Prevalence and risk factors. Cureus 10: e3269, 2018.

54. Kimura A, Sugimoto T, Kitamori K, Saji N, Niida S, Toba K and Sakurai T: Malnutrition is associated with behavioral and psychiatric symptoms of dementia in older women with mild cognitive impairment and early-stage Alzheimer's disease. Nutrients 11: E1951, 2019.

55. Doorduijn AS, van de Rest O, van der Flier WM, Visser M and de van der Schueren MAE: Energy and protein intake of Alzheimer's disease patients compared to cognitively normal controls: Systematic review. J Am Med Dir Assoc 20: 14-21, 2019.

56. Wolters FJ, Zonneveld HI, Licher S, Cremers LGM; Heart Brain Connection Collaborative Research Group, Ikram MK, Koudstaal PJ, Vernooij MW and Ikram MA: Hemoglobin and anemia in relation to dementia risk and accompanying changes on brain MRI. Neurology 93: e917-917e926, 2019.

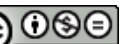

This work is licensed under a Creative Commons Attribution-NonCommercial-NoDerivatives 4.0 International (CC BY-NC-ND 4.0) License. 